\title{
Integration of IncRNA and mRNA profiles to reveal the protective effects of Codonopsis pilosula extract on the gastrointestinal tract of mice subjected to D-galactose-induced aging
}

\author{
JIE MENG ${ }^{1}$, JIAJIA LIU ${ }^{1}$, DONGMEI CHEN ${ }^{1}$, JIACHAO KANG ${ }^{1}$, YONG HUANG ${ }^{2}$, \\ DANDAN LI ${ }^{1}$, YONGQIANG DUAN ${ }^{2}$ and JING WANG ${ }^{1,3}$ \\ ${ }^{1}$ College of Clinical Medicine, ${ }^{2}$ College of Basic Medicine and ${ }^{3}$ The Key Laboratory of \\ Traditional Chinese Herbs and Prescription Innovation and Transformation of Gansu Province, \\ Gansu University of Chinese Medicine, Lanzhou, Gansu 730000, P.R. China
}

Received April 24, 2020; Accepted December 3, 2020

DOI: $10.3892 /$ ijmm.2020.4834

\begin{abstract}
Codonopsis pilosula is a type of traditional Chinese medicine that exerts an anti-aging effect and can regulate the gastrointestinal (GI) system. The aim of the present study was to investigate the underlying molecular mechanisms responsible for the anti-aging effects of Codonopsis pilosula in the GI tract of mice with D-galactose-induced aging. First, a successful mouse model of aging was established, and Codonopsis pilosula water extract was then used for treatment. The anti-aging effects of Codonopsis pilosula on the GI tract were then detected from the perspectives of tissue structure, physiological function and cell ultrastructure. Finally, in order to explore the underlying molecular mechanisms, the expression profiles of IncRNAs and mRNAs in the stomach and intestine were examined using microarray technology. A total of 117 (41 lncRNAs and 76 mRNAs) and 168 (85 lncRNA sand 83 mRNAs) differentially expressed genes associated with the anti-aging effects of Codonopsis pilosula were identified in the stomach and intestine, respectively. Through integrated analysis of the stomach and intestine, 4 hub RNAs, including
\end{abstract}

Correspondence to: Dr Jing Wang, College of Clinical Medicine, Gansu University of Chinese Medicine, 35 Dingxi Dong Road, Chengguan, Lanzhou, Gansu 730000, P.R. China

E-mail: mj2020_123@163.com

Abbreviations: NC, normal control; AM, aging model; L-CP, low-dose Codonopsis pilosula; M-CP, medium-dose Codonopsis pilosula; H-CP, high-dose Codonopsis pilosula; H\&E staining, hematoxylin and eosin staining; MTL, motilin; VIP, vasoactive intestinal peptide; ELISA, enzyme-linked immunosorbent assay; GO, Gene Ontology; DAVID, Database for Annotation, Visualization and Integration Discovery; RT-qPCR, reverse transcription-quantitative polymerase chain reaction; KEGG, Kyoto Encyclopedia of Genes and Genomes

Key words: RNA profiling, Codonopsis pilosula, aging mice, gastrointestinal tract, D-galactose
1 lncRNA (LOC105243318) and 3 mRNAs (Fam132a, Rorc and 1200016E24Rik) were identified, which may be associated with the anti-aging effects of Codonopsis pilosula in the GI tract of aging mice. The Kyoto Encyclopedia of Genes and Genomes analysis revealed that the metabolic pathway was an important pathway underlying the anti-aging effects of Codonopsis pilosula in the GI tract. On the whole, in the present study, 4 hub RNAs associated with these effects and their regulatory networks were found in the GI tract of aging mice. In addition, the metabolic pathway was found to play an important role in these anti-aging effects in the GI tract.

\section{Introduction}

As an integral part of the digestive system, the gastrointestinal (GI) tract not only plays an essential role in the digestion and absorption of food, but also in the absorption and metabolism of medication (1). However, the digestive system experiences a series of senescence and degeneration events from structure to function during aging (2). These changes have a certain impact on the intake, digestion, absorption and utilization of nutrients in the elderly, and directly or indirectly participate in the occurrence and development of digestive diseases in these patients (3). It has been reported that digestive diseases have become the second largest disease group, following cardiovascular diseases, in hospitalized elderly patients aged $>60$ years (4). The identification of effective strategies with which to alleviate the degradation of GI structure and function plays a vital role in improving the quality of life of the elderly.

Natural aging models are time-consuming, and as a result of individual differences, the process of aging is difficult to control. D-galactose (D-gal) is a normal nutrient in the body; however, if consumed in excess, it will lead to metabolic disorder; under the action of galactose oxidase, D-gal is converted into aldose and hydrogen peroxide, thus producing a large number of superoxide anion free radicals, causing oxidative damage, which leads to aging (5). Using D-gal to model aging can speed up the aging process, shorten the study time, and the animals have a higher survival rate throughout the study period (5). Thus, the model of D-gal-induced 
aging is often used for studies of aging and anti-aging (6-8). Codonopsis pilosula is a traditional Chinese medicine and food supplement in China. Gansu Province is one of the main areas of production of Codonopsis pilosula in China (9-11). Of note, it has been reported that Codonopsis pilosula, a traditional Chinese medicine, exerts an anti-aging effect and can regulate the GI system (12). Previous studies have focused on antioxidant stress and immune regulation (13-16). However, there is still a lack of systematic research at the molecular level, considering that traditional Chinese medicine has the properties of being able to affect multiple targets and organs simultaneously (17-19). In particular, the changes in the GI tract occurring during D-gal-induced aging and Codonopsis pilosula treatment in mice from the perspective of the transcriptome remain unclear. Transcriptomics is the study of researching the expression and regulation of genes under specific conditions at the RNA level, and it has been used in a number of fields $(20,21)$. IncRNAs play a complex and precise regulatory role in gene expression (22); thus, it is necessary to integrate lncRNAs and mRNAs in order to explore the mechanisms underlying the anti-aging effects of Codonopsis pilosula at the transcriptome level.

In the present study, in order to comprehensively explore the underlying molecular mechanisms of the anti-aging effects and GI regulation by Codonopsis pilosula, a mouse model of aging was successfully established with the use of D-gal, and water extract of Codonopsis pilosula was then used for treatment intervention. The structural and functional changes of the GI tract were then observed in aging mice and in mice treated with Codonopsis pilosula. Finally, biochip technology and bioinformatics were performed to explore the targets, pathways and network involved in the anti-GI senescence effects of Codonopsis pilosula. The findings of the present study may provide basic evidence for the anti-aging and GI regulatory functions of Codonopsis pilosula.

\section{Materials and methods}

Animals. A total of 100 specific pathogen-free grade Kunming mice (50 males and 50 females; age, 2 months; body mass, $20 \pm 2 \mathrm{~g}$ ) were purchased from the Gansu University of Traditional Chinese Medicine Scientific Research Laboratory Animal Center [Lanzhou, China; animal production certificate no. SCXK (Gan) 2015-0002; animal use certificate no. SKXK (Gan) 2015-0005]. All mice were raised at the Scientific Experimental Animal Center of Gansu University of Chinese Medicine. Males and females were raised in separate cages at room temperature $\left(20-25^{\circ} \mathrm{C}\right)$, were fed with general mouse feed and natural light, and had free access to double distilled water. All experimental procedures and protocols were in accordance with the guidelines of the Institutional Animal Ethics Committee of Gansu University of Chinese Medicine (approval no. 2017-106).

Drugs and reagents. Codonopsis pilosula (Franch.) Nannf. was collected from Min County (Gansu, China), and was identified as white Codonopsis pilosula by Professor Tao Du (Teaching and Research Department of Traditional Chinese Medicine Resources, Gansu University of Traditional Chinese Medicine, Lanzhou, China). In order to control the quality of Codonopsis pilosula, the polysaccharide of Codonopsis pilosula was determined by the phenol-sulfuric acid method $(23,24)$, and the average polysaccharide content of Codonopsis pilosula in the present study was $43.2 \%$. The identified Codonopsis pilosula weighed $100 \mathrm{~g}$, and was boiled twice in water for $1 \mathrm{~h}$ each time. A total of 10-fold the amount of water was added the first time, and 6-fold the amount of water the second time. The two extracting solutions were mixed, filtered and concentrated into water decoction containing $0.5 \mathrm{~g} / \mathrm{ml}$ original medicinal material, and were then placed in the refrigerator $\left(4^{\circ} \mathrm{C}\right)$ for maintenance. The water decoction was prepared every 5 days. D-gal was purchased from Merck KGaA (lot no. 24895207) and $12 \mathrm{~g} / \mathrm{l}$ was prepared with normal saline prior to use. Enzyme-linked immunosorbent assay (ELISA) kits for D-xylose, motilin (MTL) and vasoactive intestinal peptide (VIP) were purchased from Shanghai Jianglai Industrial Limited By Share Ltd. (lot no. 20171105). Animal food was purchased from Beijing Ke'ao Xie Li Feed Co., Ltd. (lot no. 09092402).

Animal model. A total of 100 mice were randomly divided into the normal control (NC group), aging model (AM group), and Codonopsis pilosula low-, medium- and high-dose groups (L-, M- and H-CP groups, respectively) ( $\mathrm{n}=20,50 \%$ males and $50 \%$ females per group). The mice in the AM, and L-, M- and $\mathrm{H}-\mathrm{CP}$ groups received a subcutaneous injection of D-gal solution into the back of the neck at a concentration of $50 \mathrm{~g} / \mathrm{l} \mathrm{D}$-gal per mouse per day (injection volume, $0.025 \mathrm{ml} / \mathrm{g}$ ). The mice in the L-, M- and H-CP groups were intragastrically administered 5, 10 and $15 \mathrm{~g} / \mathrm{kg}$ Codonopsis pilosula solution, with the NC and the AM group receiving an equal amount of normal saline. Continuous modelling was performed for 42 days. For the preparation of the model of aging, a decrease in body weight loss and thymus index indicated the successful establishment of the model (25-27).

Determination of body weight and thymus index. The body weights of the mice in each group were measured and recorded every 2 days. After $2 \mathrm{~h}$ of the final intragastric administration, the mice were anesthetized by intraperitoneal injection with $5 \%$ chloral hydrate at a dose of $0.4 \mathrm{~g} / \mathrm{kg}$, and the abdominal cavity was then rapidly cut open, and the thymus glands were completely removed. Excess fat was then removed from the surface, washed with saline and drained using filter paper. The thymus glands were weighed using an electronic balance. The thymus index was then calculated for each mouse as follows: Thymus index $(\%)=$ thymus weight $(\mathrm{g}) /$ body weight $(\mathrm{g}) \times 100 \%$.

Hematoxylin and eosin $(H \& E)$ staining. In order to observe the histopathological changes, complete stomachs and small intestines were separated following anesthesia by an intraperitoneal injection with $5 \%$ chloral hydrate at a dose of $0.4 \mathrm{~g} / \mathrm{kg}$. The removed stomachs and intestines were then dehydrated in a series of ethanol, embedded in paraffin and cut into 3- $\mu$ m-thick slices. The slices were dewaxed, fixed with xylene and ethanol, stained with H\&E (room temperature, $15 \mathrm{~min}$ ), and then washed and sealed. The slices were observed under an Olympus CX31 microscope by an experienced pathologist $(\mathrm{YH})$, who was not aware of the experimental procedure and grouping, and the degree of glandular number decrease was 
divided into no, mild, moderate and severe grades according to the Chinese consensus on chronic gastritis (28), with corresponding scores ranging from 0 to 3 . The specific methods of classification are as follows: i) Mild: The number of innate glands decreases by not $>1 / 3$ of the original glands, leaving most of the glands intact; ii) moderate: The number of proper glands decreases by $>1 / 3$, but not by $>2 / 3$. The remaining glands are irregularly distributed; iii) Severe: The number of innate glands reduced by $>2 / 3$, with only a few glands remaining, or even completely disappeared. For each pathological slice, the observer randomly selected 5 visual fields to observe the number of glands, and scored each visual field according to the reduction of the number of glands. The average score of the 5 visual fields was used for statistical analysis. Mucosal thickness was measured under an Olympus CX31 microscope with a 20X lens objective Finally, typical images were captured computer monitor (L197WA; Lenovo).

Determination of D-xylose, MTL and VIP. Following the last intragastric administration of the Codonopsis pilosula, the mice in each group were fasted without water for $24 \mathrm{~h}$ and gavaged with 3\% D-xylose suspension at a volume of $20 \mathrm{ml} / \mathrm{kg}$. After $2 \mathrm{~h}$, the animals were anesthetized by an intraperitoneal injection with 5\% chloral hydrate at a dose of $0.4 \mathrm{~g} / \mathrm{kg}, 0.5 \mathrm{ml}$ blood was collected once from the femoral artery from each mouse, and serum was prepared to measure the D-xylose concentration. The small intestinal tissues near the gastric antrum were then rapidly cut up and cleaned to yield homogenates. The blood and tissue homogenates were centrifuged at $1,500 \mathrm{x}$ g for $10 \mathrm{~min}$ at $4{ }^{\circ} \mathrm{C}$, and the serum and supernatant were collected. ELISA was performed to detect the levels of D-xylose, MTL and VIP. First, the kits were taken out, and were maintained at room temperature for $30 \mathrm{~min}$. The standard, blank and sample holes were labelled. The samples were then added in turn, and incubated at $37^{\circ} \mathrm{C}$ for $30 \mathrm{~min}$. The plate was washed 3 times, and the excess liquid was drained. Chromogenic agent was then added and kept away from the light at $37^{\circ} \mathrm{C}$ for $15 \mathrm{~min}$. Finally, the termination solution was added to terminate the reaction. The blank holes were set to zero, the absorbance value of each hole was detected at $450 \mathrm{~nm}$ within $15 \mathrm{~min}$, and the concentration of D-xyose, MTL and VIP in the samples was calculated.

Transmission electron microscope. To observe the ultrastructural changes of the stomach and intestinal tissue cells, the mice were anesthetized by an intraperitoneal injection with $5 \%$ chloral hydrate at a dose of $0.4 \mathrm{~g} / \mathrm{kg}$, and their completed stomachs and intestines were extracted, rinsed with normal saline and dried using filter paper. The stomachs and intestines from the mice in the $\mathrm{NC}, \mathrm{AM}$ and $\mathrm{H}-\mathrm{CP}$ groups were cut into small sections of $\sim 1 \mathrm{~mm}^{3}$, which were placed into a $2.5 \%$ glutaraldehyde solution precooled at $4^{\circ} \mathrm{C}$ for overnight fixation, rinsed with PBS 3 times, and fixed with $1 \%$ osmic acid for $1 \mathrm{~h}$, then dehydrated, permeated, embedded, dried, so they could be cut into thin, 70-nm-thick slices; they were then dyed with uranyl acetate for $30 \mathrm{~min}$ (room temperature), rinsed, and then re-dyed with lead citrate for $10 \mathrm{~min}$ (room temperature). Finally, the ultrastructural changes were observed in the GI tract by transmission electron microscopy (JEM-1230; Jeol). Mice were euthanized by cervical dislocation when the following humanized endpoints were reached: Weakness (inability to eat, drink and activities). The criteria for the confirmation of death following euthanasia were the following: No breathing for 3 consecutive minutes, no heartbeat and no blinking reflex.

RNA extraction, labelling and hybridization. Total RNA containing small RNA was extracted from the stomachs and intestinal tissues from the mice in the $\mathrm{NC}, \mathrm{AM}$ and $\mathrm{H}-\mathrm{CP}$ groups ( $n=3$ ) using TRIzol reagent (Thermo Fisher Scientific, Inc.) and purified using the mirVana miRNA Isolation kit (Thermo Fisher Scientific, Inc.). The purity and concentration of the RNA were determined from OD260/280 readings using a spectrophotometer (NanoDrop ND-1000). The RNA integrity was determined using $1 \%$ formaldehyde denaturing gel electrophoresis. The CapitalBio Technology Mouse LncRNA Array V1 (4x180 K format) was performed in the present study. All microarray experiments, including labelling and hybridization, were conducted according to the manufacturer's instructions.

Microarray data analysis. The lncRNA and mRNA array data were analyzed for data summarization, normalization and quality control using the GeneSpring software V13.0 (Agilent Technologies, Inc.). The threshold values of Ifold changel $\geq 1.5$ and a Benjamini-Hochberg corrected value of $\mathrm{P} \leq 0.05$ were used to select the differentially expressed genes. The data were Log2-transformed and median-centered by genes using the Adjust Data function of CLUSTER 3.0 software (http://bonsai. hgc.jp/ mdehoon/software/cluster/). Finally, the Java Treeview (Stanford University School of Medicine, Stanford, USA) was performed to tree visualization. Total RNA microarray data were uploaded onto the GEO database (http://www.ncbi.nlm. nih.gov/geo/; accession no. GSE140949). Please note that as the GSE140949 database is confidential and will be release on December 31, 2020.

Kyoto Encyclopedia of Genes and Genomes (KEGG) and Gene Ontology $(G O)$ enrichment analysis. In order to examine the biological functions of the mRNAs in the stomach and intestine related to the anti-aging effects of Codonopsis pilosula, the common differentially expressed mRNAs between the AM vs. $\mathrm{NC}$ group and the H-CP vs. AM group, were selected in the stomach and intestine, respectively. KOBAS 3.0 (http://kobas. cbi.pku.edu.cn/) was used to perform KEGG pathway enrichment analysis and David 6.8 (https://david.ncifcrf.gov/) was used to perform $\mathrm{GO}$ enrichment analysis for the mRNAs related to the anti-aging effects of Codonopsis pilosula in the stomach and intestine, respectively. KEGG pathways and GO terms were considered significantly enriched with at a value of $\mathrm{P}<0.05$.

Construction of IncRNA-mRNA co-expression network in the stomach and intestine. IncRNA has the ability to regulate the expression of mRNAs. In the present study, lncRNA-mRNA co-expression networks were constructed to investigate the functions of lncRNAs and to reveal the regulatory associationb between lncRNAs and mRNAs in the stomach and intestine. In order to determine the contribution of the IncRNA-mRNA co-expression network, pairwise Pearson's correlation coefficients between lncRNAs and mRNAs were calculated using their expression levels. Only 
Table I. Sequences of primers used for RT-qPCR in the present study.

\begin{tabular}{lll}
\hline Gene & Direction & Primer sequence 5' $\rightarrow 3^{\prime}$ \\
\hline GAPDH & Forward & GTTGTCTCCTGCGACTTCA \\
GAPDH & Reverse & TGGTCCAGGGTTTCTTACTC \\
Fam132a & Forward & GGCCTTCTACTGCCGTTTGA \\
Fam132a & Reverse & TCCTTGCAGTTCCACCAGAG \\
$1200016 \mathrm{E} 24$ Rik & Forward & AGGTCCATTTGGGTTGGTCA \\
$1200016 \mathrm{E} 24$ Rik & Reverse & TACAGGGCAAGTGCGATTTC \\
RORC & Forward & TGCCTGTTTCTGGGACCTA \\
RORC & Reverse & TATCTCCACCCCACCCTCATT \\
LOC105243318 & Forward & AGGTCCATTTGAGTTGGTCGT \\
LOC105243318 & Reverse & CTGAGGATACGCAATTTCCAG
\end{tabular}
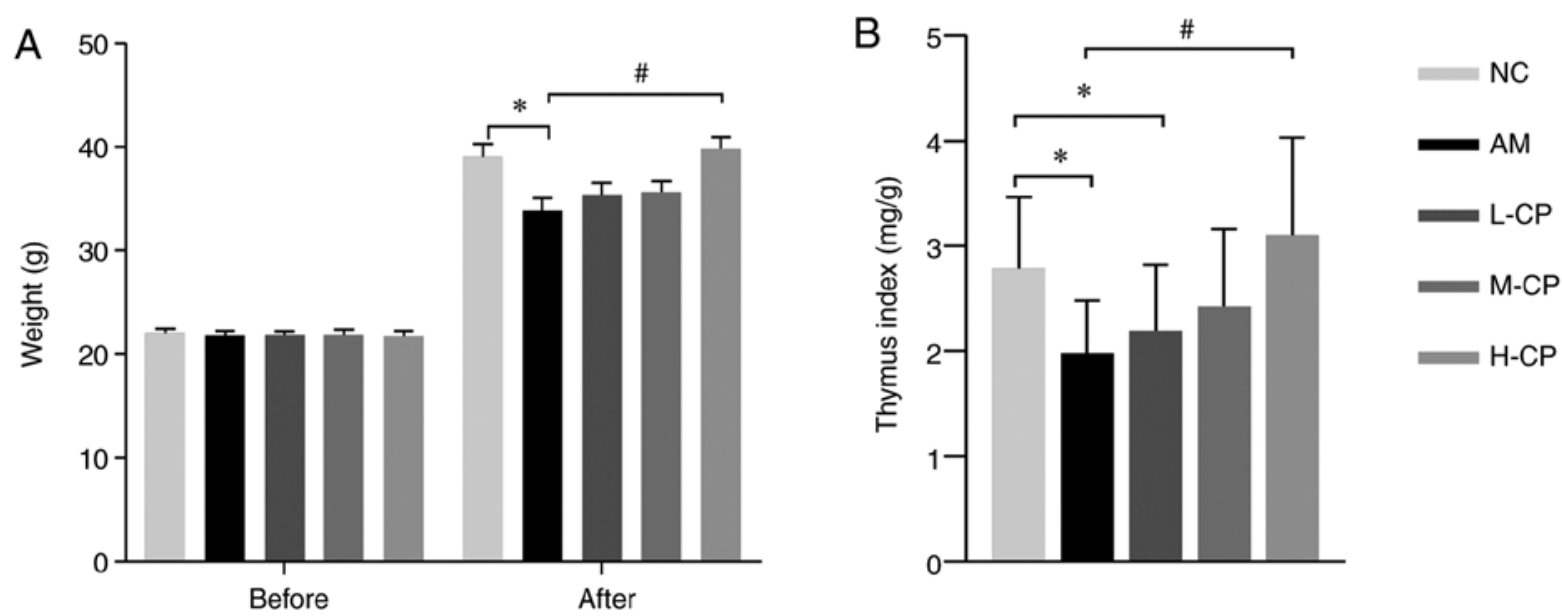

Figure 1. Effect of Codonopsis pilosula on aging mice ( $\mathrm{n}=10)$. (A) Body weight before and after the experiment. (B) Thymus index. " $\mathrm{P}<0.05 \mathrm{vs}$. NC group;

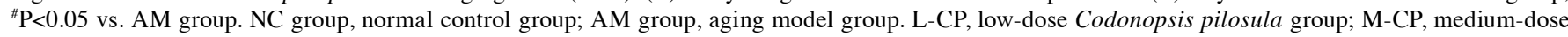
Codonopsis pilosula group; H-CP group, high-dose Codonopsis pilosula group.

the pairs with $|\mathrm{R}|>0.85$ and $\mathrm{P}<0.05$ were considered relevant and used to contribute to the co-expression network in the stomach and intestine, respectively. Finally, these networks were visualized using Cytoscape 3.8.1 (https://www.bytesin. com/software/Cytoscape/).

Real time-quantitative polymerase chain reaction (RT-qPCR). Stomach and intestinal tissue from the NC, AM and H-CP groups were homogenized in $1 \mathrm{ml}$ TRIzol reagent. Total RNA was isolated according to the manufacturer's instructions. SYBR-Green PCR Master Mix (Arraystar) was used for qPCR analysis, and all reactions were run on a QuantStudio TM 7 Flex Real-time PCR System (Thermo Fisher Scientific, Inc.) using the following steps: Denaturation step $\left(95^{\circ} \mathrm{C}\right.$ for $\left.10 \mathrm{~min}\right)$, and 45 cycles of three step amplification (denaturation, $95^{\circ} \mathrm{C}$ for $10 \mathrm{sec}$; annealing, $58^{\circ} \mathrm{C}$ for $5 \mathrm{sec}$; and extension, $72^{\circ} \mathrm{C}$ for $10 \mathrm{sec})$. All RNA expression data were normalized to GAPDH. The relative expression of the genes was calculated using the $2^{-\Delta \Delta \mathrm{Cq}}$ method (29). The primers used in the present study are summarized in Table I.

Statistical analysis. Data were analyzed using SPSS 24.0 statistical software, and expressed as the means \pm standard deviation. Based on the Shapiro-Wilk normality test, one-way ANOVA was used to analyze the differences among groups, and Tukey's post hoc test was then used to correct multiple comparisons. A P-value $<0.05$ was considered to indicate a statistically significant difference.

\section{Results}

Codonopsis pilosula intervention can improve weight and thymus index in aging mice. As shown in Fig. 1A, no significant differences in body weight were observed among the groups prior to the experiment. However, following the experiment, compared with the $\mathrm{NC}$ group, the body weights of the mice in the AM group decreased significantly, with a maximum body weight loss of $20 \%$ [maximum body weight loss $=($ maximum body weight in NC group-minimum body weight in AM group)/the maximum body weight in $\mathrm{NC}$ group: (40.8-32.6)/40.8 x100] and an average weight loss of $13 \%$. Of note, a significant increase in body weight was observed in the $\mathrm{H}-\mathrm{CP}$ group, as compared with the AM group. A significantly different diversity was observed in the thymus index level in the AM and L-CP groups, as compared with the NC group, and in the H-CP group, as compared with the AM group (Fig. 1B). 

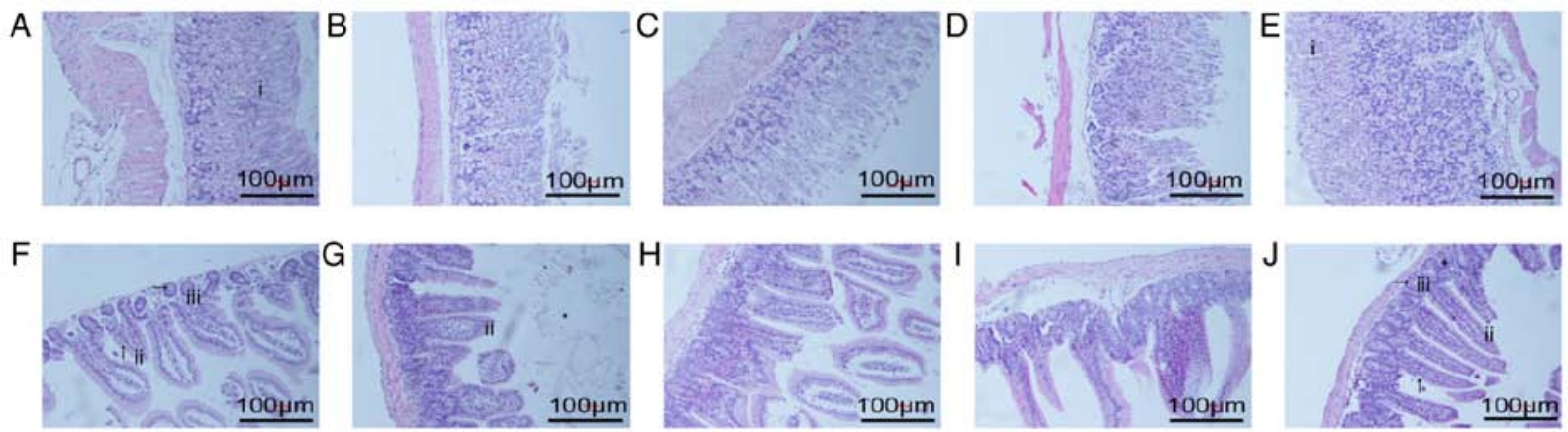

Figure 2. Effect of Codonopsis pilosula on gastrointestinal structure (hematoxylin and eosin staining; magnification, x10). (A-E) Light microscopy of stomachs from the NC, AM, and L-, M- and H-CP groups. (F-J) Light microscopy of intestines from the NC, AM, and L-, M- and H-CP groups. i, fundic gland; ii, intestinal villus; iii, small intestinal gland. Rightward arrows $(\rightarrow)$ indicate Paneth cells. Upward arrows $(\uparrow)$ indicate absorptive cells. MTL, motilin; VIP, vasoactive intestinal peptide; NC group, normal control group; AM group, aging model group; L-CP, low-dose Codonopsis pilosula group; M-CP, medium-dose Codonopsis pilosula group; H-CP group, high-dose Codonopsis pilosula group.
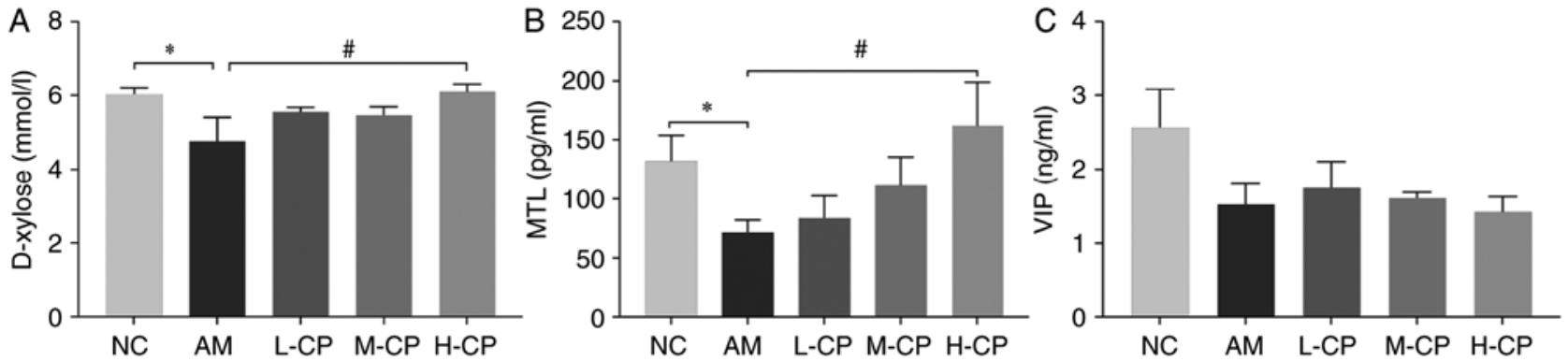

Figure 3. Effect of Codonopsis pilosula on gastrointestinal tract function (n=10). (A) D-xylose, (B) motilin and (C) vasoactive intestinal peptide levels in all groups. ${ }^{*} \mathrm{P}<0.05$ vs. $\mathrm{NC}$ group; ${ }^{\#} \mathrm{P}<0.05$ vs. AM group. NC group, normal control group; AM group, aging model group; L-CP, low-dose Codonopsis pilosula group; M-CP, medium-dose Codonopsis pilosula group; H-CP group, high-dose Codonopsis pilosula group.

These results revealed that Codonopsis pilosula can ameliorate weight loss and thymus index reduction induced by aging in the mouse model of aging.

Codonopsis pilosula can protect GI tissue structure in the aging mice. In the stomach, the fundic glands were regular in shape and abundant in quantity in the NC group (Fig. 2A). Compared with the NC group, the deep glands and gastric mucosa of the AM group were atrophic to a certain extent (Fig. 2B). The results of the L-, M- and H-CP groups are shown in Fig. 2C-E, respectively. The fundus glands and mucosa in the $\mathrm{M}$ group and the $\mathrm{H}-\mathrm{CP}$ group were intact and of regular shape (Fig. 2D and E). The exact number of gastric mucosa thickness and inherent stomach glands are presented in Table SI. The results revealed that the medium and high dose of Codonopsis pilosula reversed the changes of gastric histomorphology in aged mice.

In the intestine, the intestinal mucosa of the mice was complete with regular arrangement of intestinal villi and abundant glands in the NC group (Fig. 2F). The intestinal mucosal tissues and structures were damaged, atrophic and sparsely arranged in the AM group (Fig. 2G). As compared with the AM group, these damages were not improved in either the L- or M-CP groups (Fig. 2H and I). However, this was markedly ameliorated in the $\mathrm{H}-\mathrm{CP}$ group (Fig. 2J); the intestinal mucosal tissue and structural damage was reduced, and the arrangement was more orderly. These results demonstrated that the high dose of Codonopsis pilosula exerted protective effects on the intestinal structure in aging mice.
Codonopsis pilosula can enhance D-xylose absorption, as well as MTL and VIP secretion in the GI tract in aging mice. Three indexes were used to determine the digestive function diversification in the present study, including D-xylose, MTL and VIP (Fig. 3). As compared with the NC group, the D-xylose level was markedly decreased in the AM group. As compared with the AM group, the D-xylose level was significantly increased in the H-CP group. The MTL level in the mice in the AM group was considerably decreased, as compared with the NC group, and it was significantly increased in the $\mathrm{H}-\mathrm{CP}$ group as compared with the AM group. These results demonstrated that Codonopsis pilosula promoted D-xylose absorption and increased MTL secretion in the GI tract; the higher the dose, the more significant the effect. The VIP level in the AM group was decreased as compared with the NC group. The L-CP group exhibited the largest change in VIP levels as compared with the AM group, followed by the M-CP group; the VIP level gradually decreased as the concentration of the Codonopsis pilosula water extract increased. This result indicated that the low of dose Codonopsis pilosula stimulated VIP secretion; however, these changes were not statistically significant $(\mathrm{P}>0.05)$.

Codonopsis pilosula protects the GI tissue cell ultrastructure in aging mice. According to Figs. 1, 2 and 3A and B, it was found that compared with the L-CP and M-CP groups, the $\mathrm{H}-\mathrm{CP}$ group exhibited the most significant anti-aging effect 

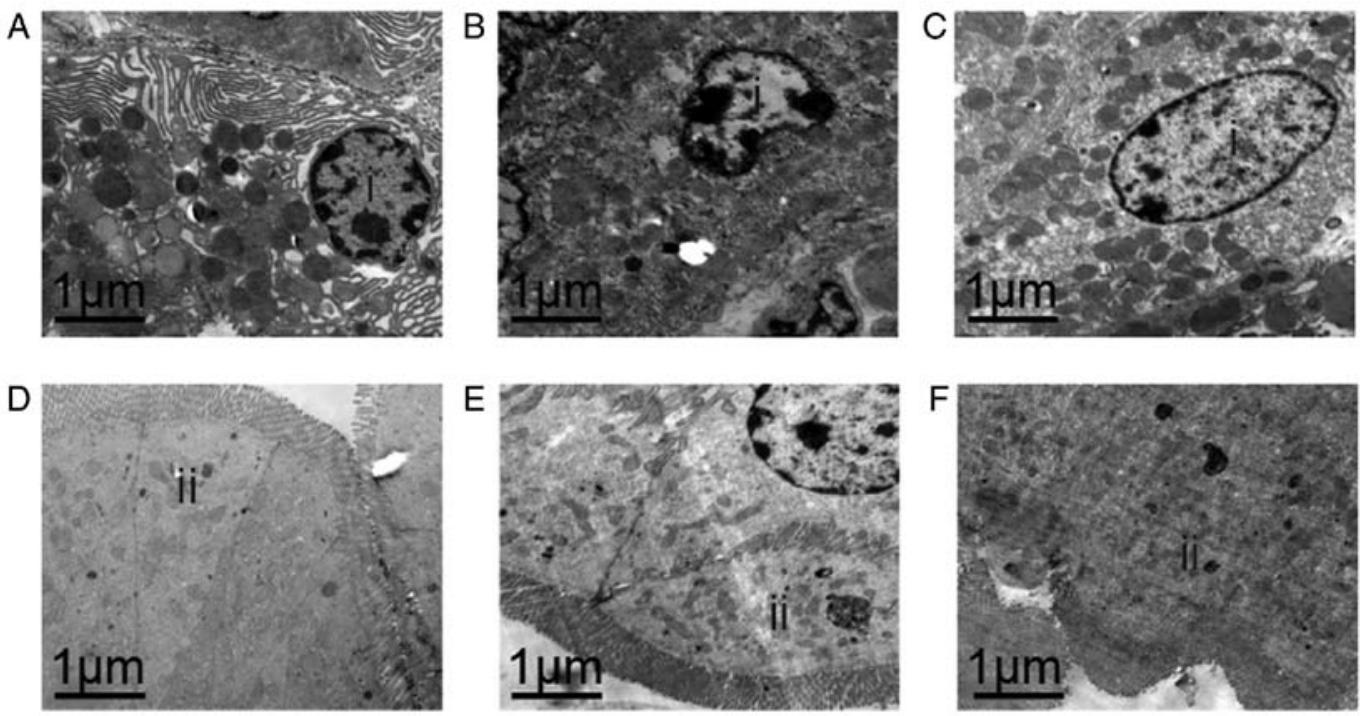

Figure 4. Ultrastructural changes in gastrointestinal tissue (magnification, x10,000). Electron microscopy of the normal control group, aging model and high-dose Codonopsis pilosula groups in the (A-C) stomach and (D-F) intestine. i, zymogenic cell; ii, absorptive cell.

in the gastrointestinal tract of the mice (in the H-CP group, body weight and thymus index increased most evidently, and the improvement of pathological damage was the most evident D-xylose absorption and MTL secretion were also were the highest), therefore, we choose H-CP for our subsequent transmission electron microscope. In the stomach, zymogenic cells exhibited a normal structure in the NC group (Fig. 4A). However, organelle lysis and structure destruction were observed in zymogenic cells in the AM group (Fig. 4B). However, as compared with the AM aging model group, the structure of the zymogenic cells appeared to be improved (almost normal) in the H-CP group (Fig. 4C).

In the intestine, normal intestinal epithelial cells with a free surface were observed; the cytoplasm of the intestine absorbing cells and the whole intestinal absorbing cells were also observed in the NC group (Fig. 4D). As compared with the NC group, no marked changes were observed in the AM group, apart from a certain degree of autophagy (Fig. 4E). There was no damage to the intestinal epithelial cells, and organelle changes were not evident in the H-CP group (Fig. 4F). In combination, these results suggested that the high dose of Codonopsis pilosula had the ability to protect the GI tissue cell ultrastructure in aging mice to a certain extent.

IncRNA and mRNA profiling in the stomach of aging mice treated with Codonopsis pilosula. In the stomach, 359 and 1,552 differentially expressed lncRNAs were identified between the AM and NC groups, and between the $\mathrm{H}-\mathrm{CP}$ and AM groups. A total of 743 and 1,106 significantly altered mRNAs were identified between the $\mathrm{AM}$ and $\mathrm{NC}$ groups, and between the $\mathrm{H}-\mathrm{CP}$ and $\mathrm{AM}$ groups (IFold changel $\geq 1.5 ; \mathrm{P}<0.05$; Fig. 5A and B). Lists of the top 50 up- and downregulated lncRNAs and mRNAs in the stomachs from the mice in the $\mathrm{AM}$ vs. the $\mathrm{NC}$ groups, and $\mathrm{H}-\mathrm{CP}$ vs. the $\mathrm{AM}$ groups are presented in Table SII.

In order to reveal which RNAs were involved in the anti-aging effects of Codonopsis pilosula, the common differentially expressed IncRNAs and mRNAs between the AM vs. $\mathrm{NC}$ group and the $\mathrm{H}-\mathrm{CP}$ vs. AM group, were selected as the RNAs involved in the anti-aging effects of Codonopsis pilosula in the stomach (Fig. 5C and D). A total of 117 RNAs (41 lncRNAs and 76 mRNAs) associated with the anti-aging effects of Codonopsis pilosula in the stomach were identified, and the details are presented in Table SIII.

IncRNA and mRNA profiling in the intestine of aging mice treated with Codonopsis pilosula. In the intestine, 351 and 371 differentially expressed lncRNAs were identified between the $\mathrm{AM}$ and $\mathrm{NC}$ groups, and between the H-CP and AM groups. A total of 272 and 504 significantly altered mRNAs were identified between the AM and $\mathrm{NC}$ groups, and between the $\mathrm{H}-\mathrm{CP}$ and AM groups (IFold changel $\geq 1.5 ; \mathrm{P}<0.05$; Fig. $6 \mathrm{~A}$ and $\mathrm{B}$ ). Lists of the top 50 up- and downregulated lncRNAs and mRNAs were identified in intestines from the AM vs. NC group, and those from the $\mathrm{H}-\mathrm{CP}$ vs. AM groups are presented in Table SIV.

In order to discover which RNAs were involved in the anti-aging effects of Codonopsis pilosula, the common differentially expressed lncRNAs and mRNAs between the AM vs. $\mathrm{NC}$ group and the $\mathrm{H}-\mathrm{CP}$ vs. AM group, were found to be the RNAs associated with the anti-aging effects of Codonopsis pilosula in the intestine (Fig. 6C and D). There were 168 RNAs (85 lncRNAs and 83 mRNAs) associated with the anti-aging effects of Codonopsis pilosula in the intestine (Table SV).

KEGG pathway and GO terms function analysis for differentially expressed Codonopsis pilosula anti-aging $m R N A s$ in the stomach and intestine. As shown in Fig. 7A and B, 24 and 23 significant KEGG pathways were found in the stomach and intestine, respectively, with the metabolic pathway being the common pathway between the two sites. As shown in Fig. 7C and D, the significant GO terms included biological processes, cellular components and molecular functions in the stomach and intestine. Intracellular membrane-bound organelle was the common GO term between the stomach and intestine. 
A

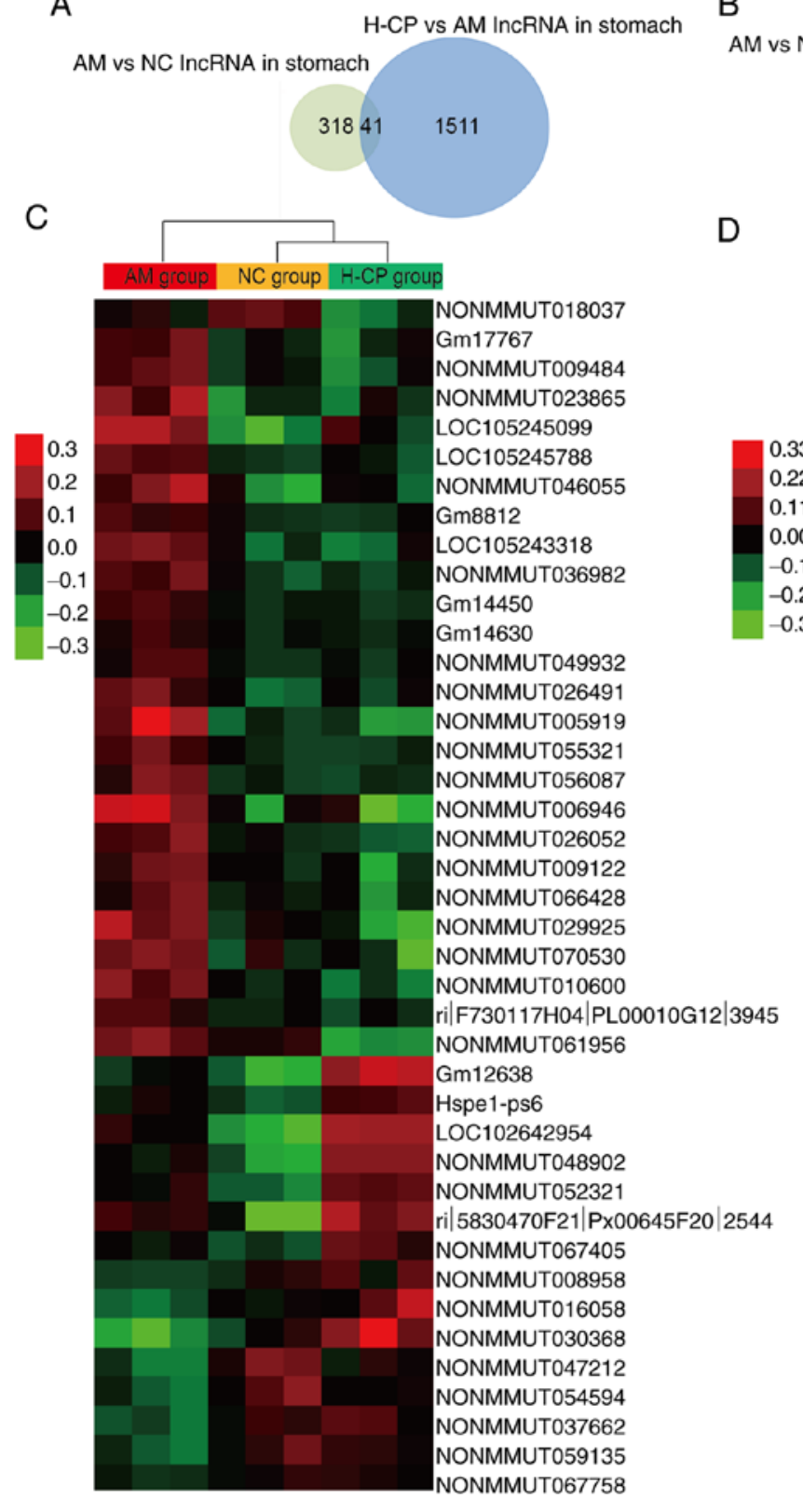

в AM vs NC mRNA in stomach $\mathrm{H}-\mathrm{CP}$ vs AM mRNA in stomach
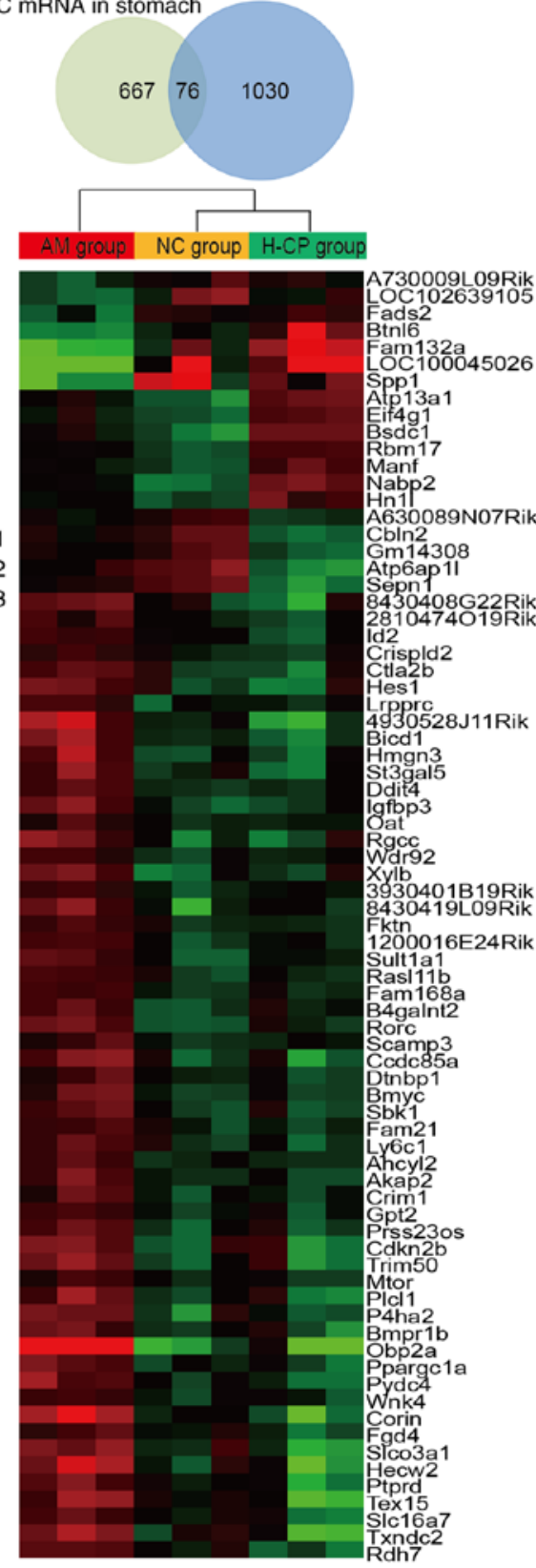

Figure 5. IncRNA and mRNA profiling in the stomach of aging mice treated with Radix Codonopsis. (A and B) Venn plot of differentially expressed lncRNAs and mRNAs between the AM and NC groups, and between the H-CP and AM groups in the stomach, respectively. (C and D) Heatmap of lncRNAs and mRNAs related to the anti-aging effects of Codonopsis pilosula in the stomach. The upregulated genes are marked in red and the downregulated genes in green. NC group, normal control group; AM group, aging model group; H-CP group, high-dose Codonopsis pilosula group.

IncRNA-mRNA co-expression network construction of the anti-aging effects of Codonopsis pilosula in the stomach, intestine and GI tract. IncRNA-mRNA co-expression networks for the lncRNAs and mRNAs related to the anti-aging effects of Codonopsis pilosula were constructed by Pearson's correlation coefficient in the stomach and intestine to explore the function of the differently expressed lncRNAs (Fig. 8A and B). To reveal the precise molecular mechanisms of the anti-aging effects of Codonopsis pilosula in the GI tract, the co-expression networks in the stomach and intestine were merged, and a sub-network, which participated in the anti-aging effects of Codonopsis pilosula in the GI tract, was then found (Fig. 8C), including 4 nodes ( 1 lncRNA and 3 mRNAs) and 3 edges.
$R T-q P C R$ verification of gene expression for the RNAs related to the anti-aging effects of Codonopsis pilosula in the GI tract. RT-qPCR was performed on 4 genes from the Codonopsis pilosula anti-aging lncRNA-mRNA co-expression network in the GI tract (Fam132a, 1200016E24Rik, RORC and LOC105243318). The expression of Fam132a was increased in the H-CP group, as compared with the AM group, and decreased in the AM group, as compared with the NC group (Fig. 9A). 1200016E24Rik, RORC and LOC105243318 were found to be decreased in the H-CP group, as compared with the AM group, and increased in the AM, as compared with the NC group (Fig. 9B-D). These results were consistent with those of the microarray expression profiling data. 
A

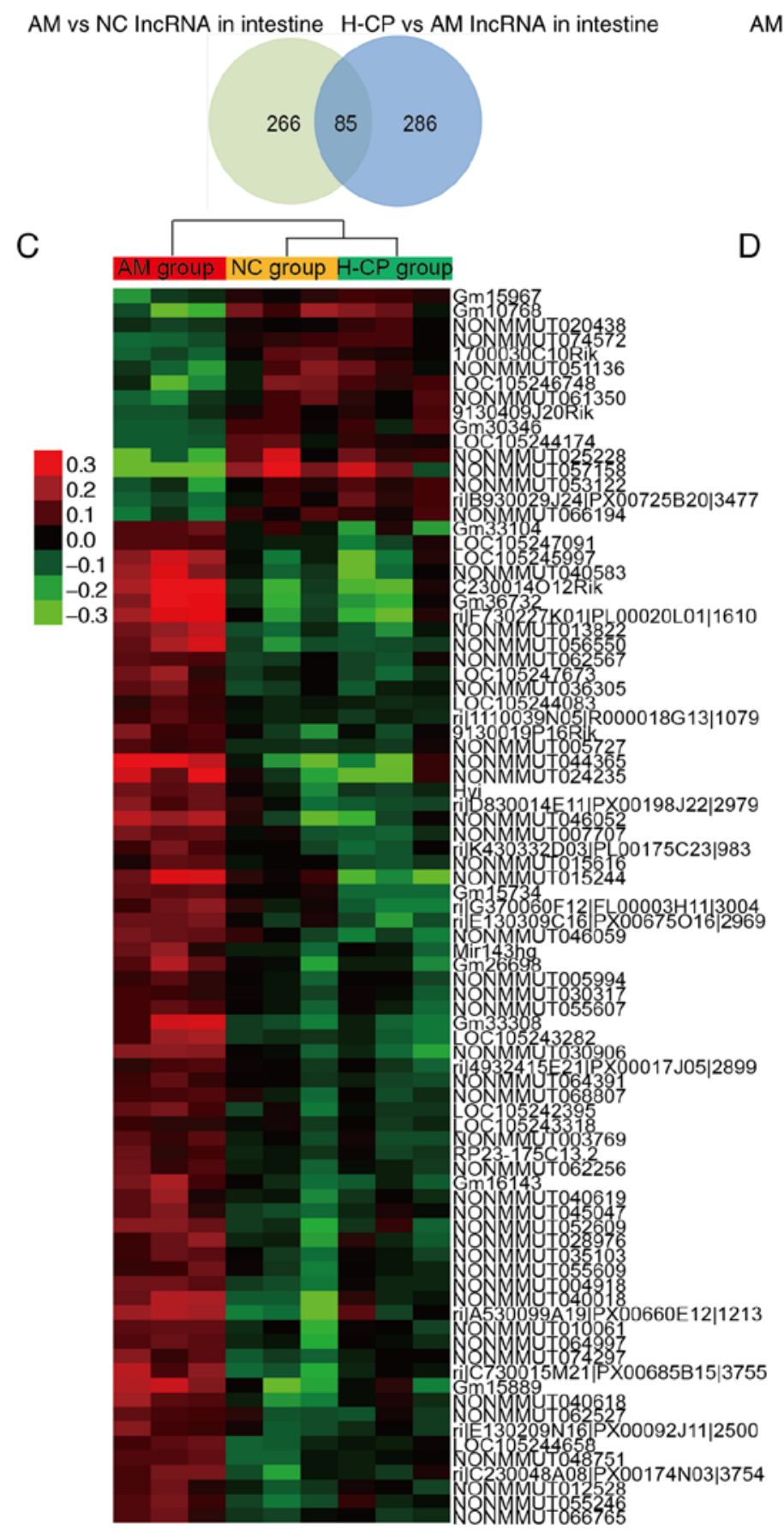

B

AM vs NC mRNA in intestine $\mathrm{H}-\mathrm{CP}$ vs $\mathrm{AM}$ mRNA in intestine

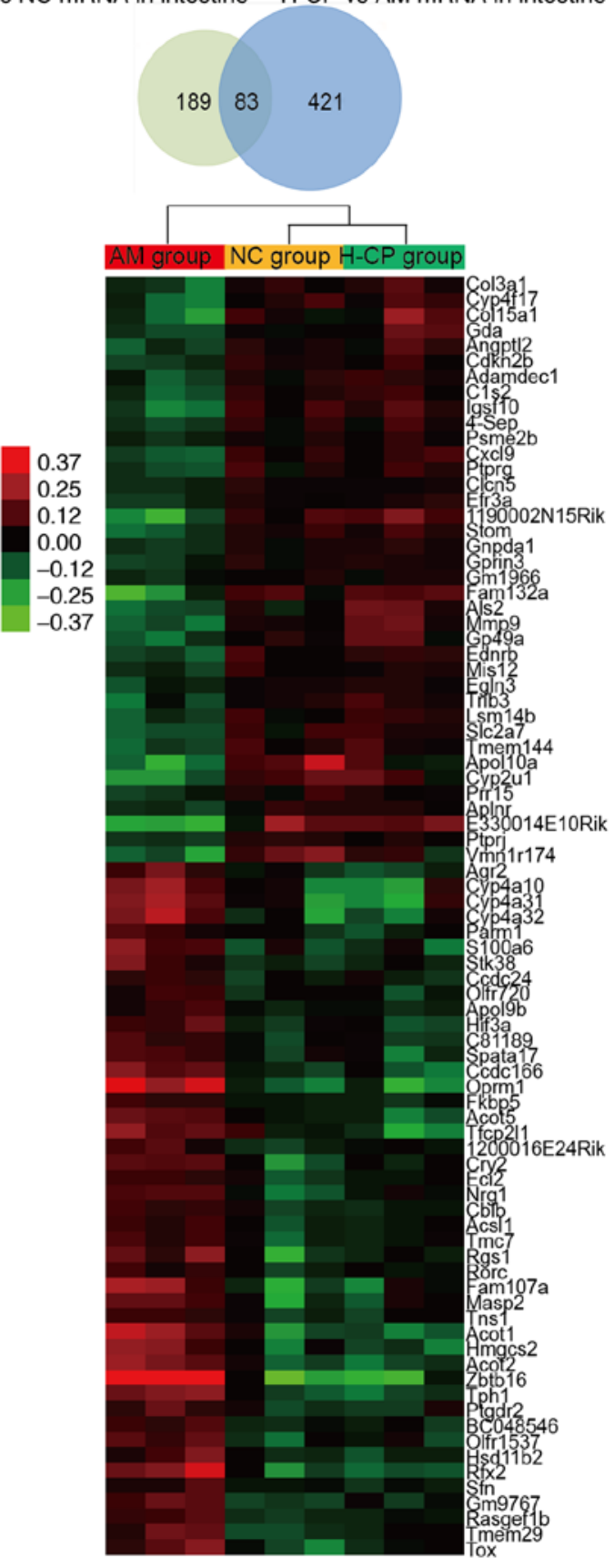

Figure 6. IncRNA and mRNA profiling in the intestine of aging mice treated with Radix Codonopsis. (A and B) Venn plot of differentially expressed lncRNAs and mRNA between the AM and NC groups, and between the H-CP and AM groups in the intestine, respectively. (C and D) Heatmap of lncRNAs and mRNAs related to the anti-aging effects of Codonopsis pilosula in the intestine. The upregulated genes are marked in red and the downregulated genes in green. $\mathrm{NC}$ group, normal control group; AM group, aging model group; H-CP group, high-dose Codonopsis pilosula group.

\section{Discussion}

Biological aging is an intrinsic, complex and irreversible process that occurs in living beings. However, aging can be delayed, a fact that has attracted considerable medical attention (6). In the present study, it was confirmed that Codonopsis pilosula improved the mouse body weight and thymus index, protected the GI structure, improved GI function, and protected the ultrastructure of GI cells in aging mice. Further analyses identified possible targets, action networks and related pathways involved in the anti-aging effects of Codonopsis pilosula in the GI tract of aging mice at the molecular level.

It has been demonstrated that aging can lead to a range of changes, including muscle mass loss, weight loss and organ atrophy in varying degrees, which increase the risk of variously age-related diseases in older individuals (30). The thymus index is one of the reference indexes used to judge aging, since thymus atrophy occurs during the aging process, which is associated with the immune aging theory of the body $(31,32)$. Pathological observation can directly reflect the 
A

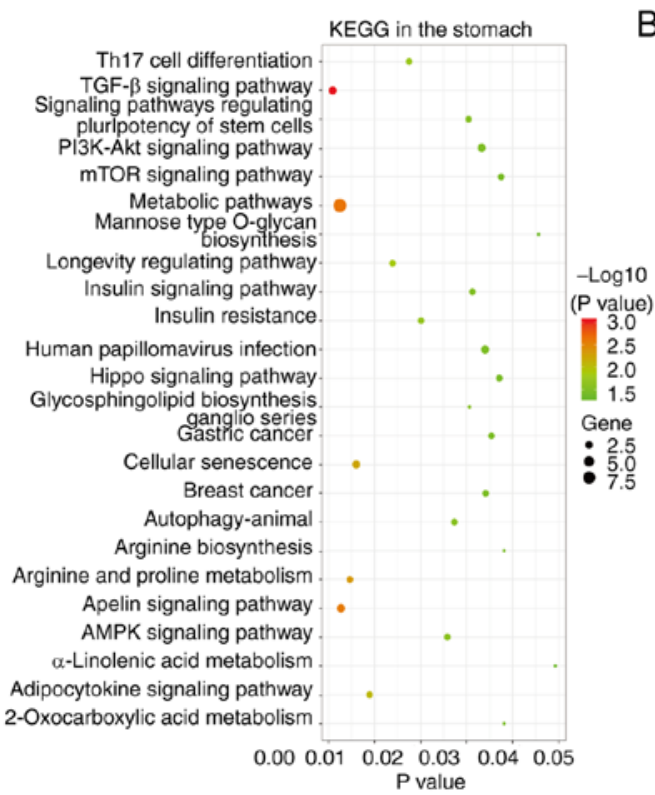

C

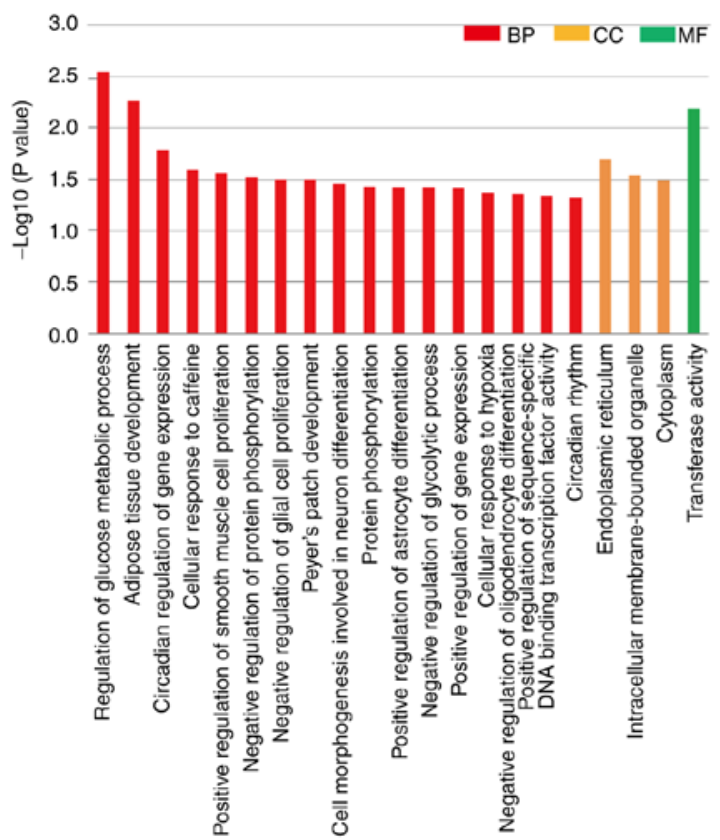

B
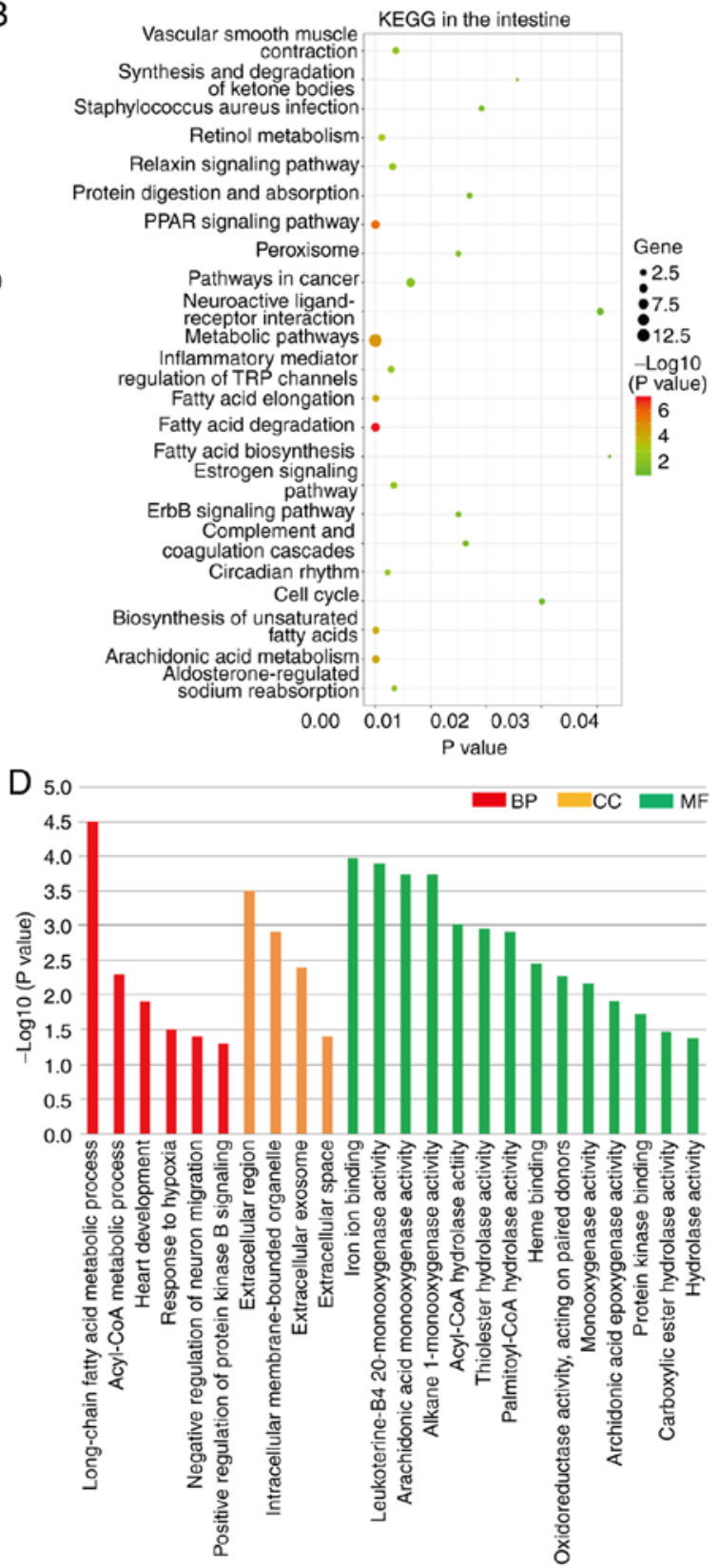

Figure 7. KEGG and GO analysis of differentially expressed mRNAs related to the anti-aging effects of Codonopsis pilosula in the stomach and intestine. (A and B) KEGG pathways for the differentially expressed mRNAs related to the anti-aging effects of Codonopsis pilosula in the stomach and intestine. (C and D) GO terms for the differentially expressed mRNAs related to the anti-aging effects of Codonopsis pilosula in the stomach and intestine. KEGG, Kyoto Encyclopedia of Genes and Genomes; TGF- $\beta$, transforming growth factor- $\beta$; PI3K, phosphatidylinositol-3-kinase; mTOR, mammalian target of rapamycin.

histomorphological changes, which can evaluate the pathological changes of the body (33). During the aging process, tissues and organs undergo a series of special pathological changes in the GI tract, including atrophy and damage of the GI mucosa (34-36), which destroys the integrity of GI mucosa structure and function, and is also closely associated with the occurrence of several aging-related diseases $(37,38)$. In the present study, the body weights and thymus indexes were decreased in the aging mice, and the mucosa of the GI tract of the aging mice shrank and was destroyed; however, all these effects were alleviated following intervention with Codonopsis pilosula, indicating that Codonopsis pilosula attenuated aging and protected the intact GI structure in aging mice.
Structural changes often lead to functional alterations. In the present study, the blood concentration of D-xylose was measured to evaluate the absorption function of the small intestine (39), which is associated with the integrity of the GI tract $(40,41)$. The concentration of MTL and VIP were measured to determine the motor and digestion functions of the GI tract. It has been reported that the expression of MTL receptor mRNA in different regions of the digestive tract decreases with age (42), and the level of VIP has been shown to be associated with several aging-related diseases $(43,44)$, particularly GI diseases. The impaired digestive capacity of the GI tract is often caused by an impaired cell secretion capacity (45). The gastric chief cell is a mature type of cell 
A

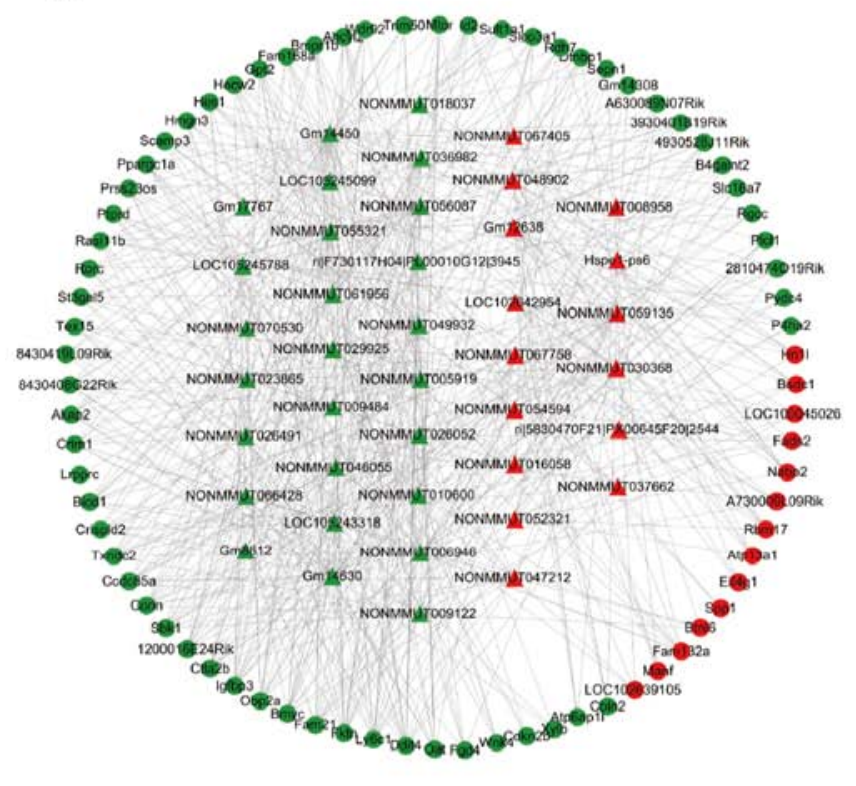

B

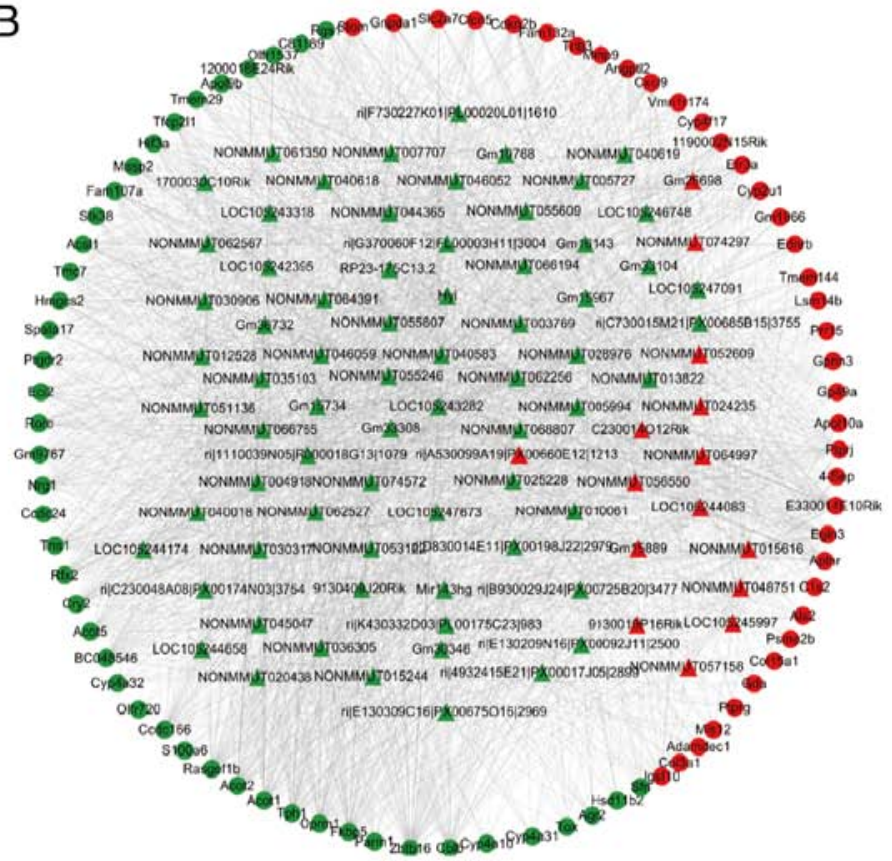

C

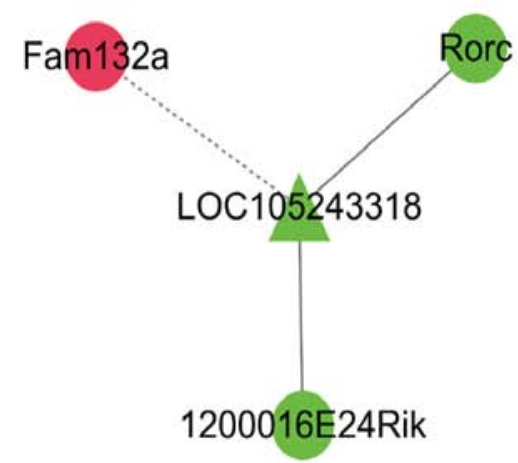

Figure 8. Codonopsis pilosula anti-aging-related lncRNA-mRNA co-expression networks. (A and B) lncRNA-mRNA co-expression network of the anti-aging effects of Codonopsis pilosula in the stomach and intestine. (C) The sub-network of of the anti-aging effects of Codonopsis pilosula in the gastrointestinal tract. The upregulated genes are marked in red and the downregulated genes in green following Codonopsis pilosula intervention. The triangle represents IncRNAs and the circle mRNAs. The solid line represents a positive correlation and the dotted line a negative correlation.

that secretes digestive enzymes, and aging causes degenerative changes in the gastric chief cells (46). Small intestinal absorption cells are involved in the digestion and absorption of sugars and lipids; the aging adult Drosophila intestine has obvious characteristics, such as a multilayer of absorptive cells and a wrong expression of cell type-specific genes (47). In the present study, the levels of D-xylose, MTL and VIP were decreased, and the ultrastructure of the gastric chief cells and small intestinal absorption cells was destroyed in the GI tract of aging mice. These results demonstrated that Codonopsis pilosula has the ability to regulate the GI function and protect cell ultrastructure in the GI tract of aging mice.

To comprehensively explore the underlying molecular mechanisms of the anti-aging effects of Codonopsis pilosula in the GI tract of aging mice, the stomach and intestine were integrated, and 4 hub RNAs were identified, including 1 lncRNA (LOC105243318) and 3 mRNAs (Fam132a, RORC and 1200016E24Rik). LOC105243318 is a predicted non-coding RNA located in the negative strand of chromosome 8 with a length of $443 \mathrm{bp}$, which begins with $3,594,757$ and ends with $3,595,409$. In the present study, it was found to be able to negatively regulate Fam 132a and positively regulate RORC and 1200016E24Rik, which may play an important role in the anti-GI senescence of Codonopsis pilosula. Fam132a is located in chromosome 4, and encodes a newly identified insulin-sensitizing adipokine, which has anti-inflammatory and glucose-lowering properties (48). In the present study, Fam132a was downregulated in aging mice and upregulated following Codonopsis pilosula treatment by the regulation of LOC105243318. RORC is the upstream regulatory gene of Th17 and Treg cells (49). Altering Th17 cytokine production during the inflammatory response in the elderly is a novel method for maintaining a healthy level of aging (50). Herein, RORC was upregulated following aging, and downregulated following Codonopsis pilosla intervention; therefore, the anti-aging effects of Codonopsis pilosula in GI tract senescence may be associated with the regulation of Th17 and Treg cells by RORC. Similar to RORC, 1200016E24Rik was positively regulated by IncRNA LOC105243318, upregulated in the GI tract of aging mice and downregulated following Codonopsis pilosula intervention. 
A
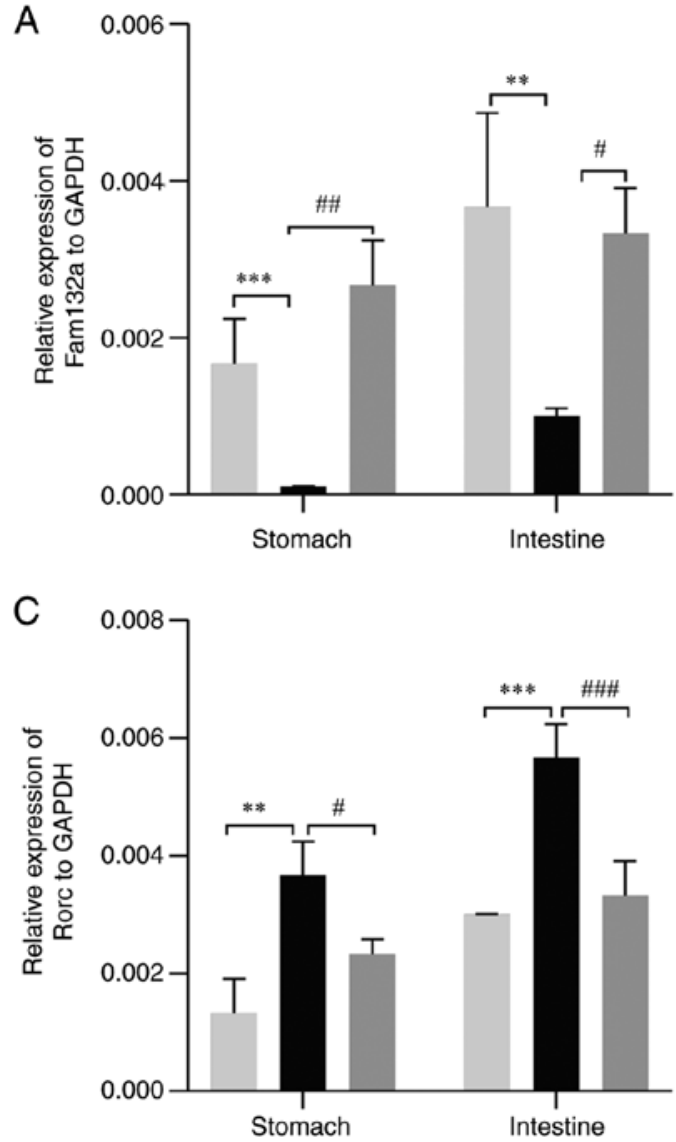

B
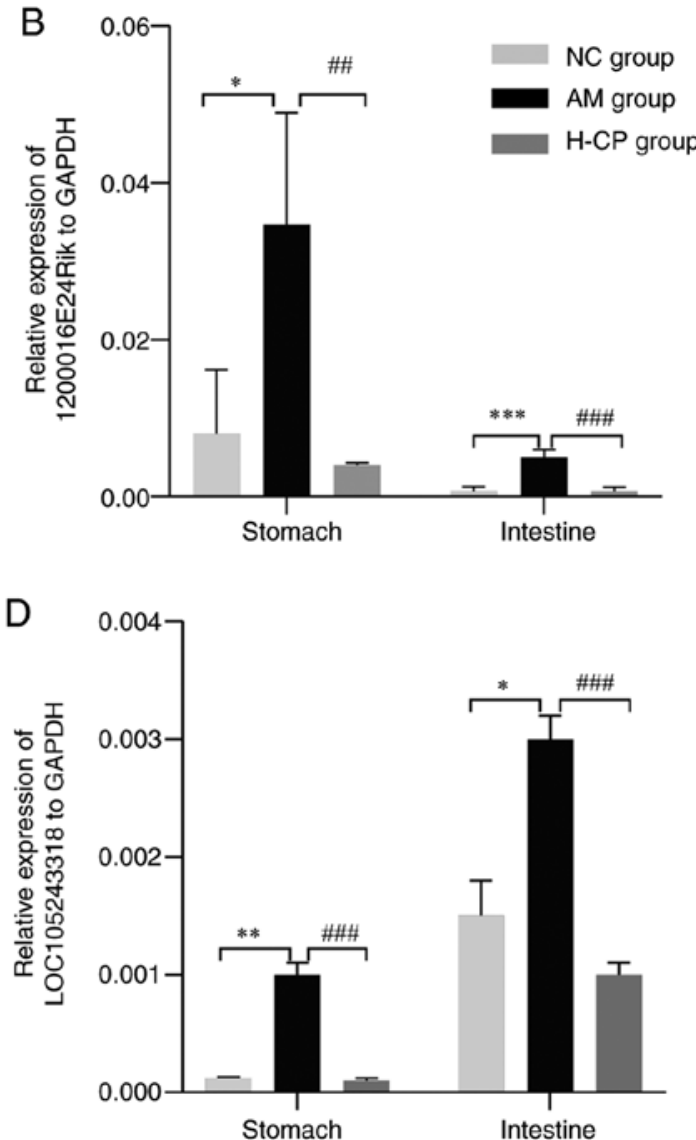

Figure 9. Results of RT-qPCR for gene expression. (A-D) The expression of Fam132a, 1200016E24Rik, RORC and LOC105243318 was detected by RT-qPCR. Data are presented as the means \pm SD. $n=3$. ${ }^{*} \mathrm{P}<0.05,{ }^{* *} \mathrm{P}<0.01$ and ${ }^{* * *} \mathrm{P}<0.001$ vs. NC group; ${ }^{\#} \mathrm{P}<0.05,{ }^{\# \#} \mathrm{P}<0.01$ and ${ }^{\# \# \#} \mathrm{P}<0.001$ vs. AM group). NC group, normal control group; AM group, aging model group; $\mathrm{H}-\mathrm{CP}$ group, high-dose Codonopsis pilosula group; RT-qPCR, reverse transcription-quantitative polymerase chain reaction.

In the present study, the metabolic pathway was found to be an important pathway playing a vital role in the ageing process of the GI tract of mice and the anti-aging effects of Codonopsis pilosula. An overall metabolic stability decline has been reported in elderly organisms (51), with an association observed between these disorders and intestinal microbiota imbalance (52). The regulation of the intestinal microbiome has become an effective therapeutic tool against age-related chronic diseases (53). Certain studies have indicated that Codonopsis pilosula polysaccharide, one of the main components of Codonopsis pilosula, can be used as a prebiotic to regulate intestinal microbiota $(54,55)$. The findings of the present study support this point; the specific underlying mechanism warrant further investigation in future studies.

In conclusion, the protective effects of Codonopsis pilosula in senescence in the GI tract were confirmed from the perspectives of tissue morphology, GI function and cell ultrastructure. A total of 4 hub RNA molecules (LOC105243318, Fam132a, RORC and 1200016E24Rik) and their regulatory association network were found to be associated with the anti-aging effects of Codonopsis pilosula in the GI tract of aging mice. The metabolic pathway may play a vital role in the process of aging and in the anti-aging effects of Codonopsis pilosula. These results provide important insight and directions for the future development of drugs and for the mining of the mechanisms of aging intervention.

\section{Acknowledgements}

The authors would like to thank Mr. Junjun Liu for providing assistance with the animal experiments, and Dr Yong Wang for assistance with the histopathological analysis.

\section{Funding}

This study was supported by the National Natural Science Foundation of China (grant nos. 81760835 and 82060829), and the General Project of Scientific Research in Colleges and Universities of Gansu Province (grant no. 2018A-052).

\section{Availability of data and materials}

The datasets used and analyzed during the current study are available from the corresponding author on reasonable request. Please note that as the data on the GEO database are confidential and will be released on December 31, 2020.

\section{Authors' contributions}

Each author has contributed significantly to the present study. JM, JW, DL, JL and YD conceived and designed the study. DL and JL performed the animal experiments. YH was responsible for the histopathological and ultrastructural observations. JM, 
DC and JK performed the statistical analyses. JM drafted the manuscript. JW and JK revised the manuscript. YD edited the original manuscript and guided the revision of the manuscript. All authors read and approved the final manuscript.

\section{Ethics approval and consent to participate}

All the experimental procedures and protocols were accordance with the guidelines of and were performed following the approval of the Institutional Animal Ethics Committee of Gansu University of Chinese Medicine (approval no. 2017-106).

\section{Patient consent for publication}

Not applicable.

\section{Competing interests}

The authors declare that they have no competing interests.

\section{References}

1. Dumic I, Nordin T, Jecmenica M, Stojkovic Lalosevic M, Milosavljevic T and Milovanovic T: Gastrointestinal tract disorders in older age. Can J Gastroenterol Hepatol 2019: 6757524, 2019.

2. Durazzo M, Campion D, Fagoonee S and Pellicano R: Gastrointestinal tract disorders in the elderly. Minerva Med 108: 575-591, 2017.

3. Zhang XL and Zheng SB: Aging of digestive system and clinic. Chin J Clin 43: 3-7, 2015 (In Chinese).

4. Zhang HD: From 2014 to 2018, a retrospective analysis was conducted on the hospitalization situation of the elderly over 60 years old in a hospital. Jiangsu Health System Management 30: 1561-1563, 2019 (In Chinese).

5. Azman KF and Zakaria R: D-Galactose-induced accelerated aging model: An overview. Biogerontology 20: 763-782, 2019.

6. Cai J, Ashraf MA, Luo L and Tang H: Effects of Codonopsis pilosula water extract on MicroRNA expression profile in D-galactose-induced senile mice. Pak J Pharm Sci 30 (Special): 1179-1183, 2017.

7. Yang J, He Y, Zou J, Xu L, Fan F and Ge Z: Effect of polygonum multiflorum thunb on liver fatty acid content in aging mice induced by D-galactose. Lipids Health Dis 18: 128, 2019.

8. Chen P, Chen F and Zhou BH: Leonurine ameliorates $\mathrm{D}$-galactose-induced aging in mice through activation of the Nrf2 signalling pathway. Aging (Albany NY) 11: 7339-7356, 2019.

9. Wang HZ, Lian ZX, Lu GD, Huang YF, Cui ZJ, Li JT and Du T: Relationship between seedling grade of Codonopsis pilosula and yield and quality of medicinal materials. Zhongguo Zhong Yao Za Zhi 41: 3950-3955, 2016 (In Chinese).

10. Zhang JQ, Su X, Wu Q, Ding SS and Sun K: Analysis of RAPD on medicinal plants of Codonopsis pilosula. Zhong Yao Cai 29: 417-420, 2006 (In Chinese).

11. Xie Q, Sun Y, Cao L, Chen L, Chen J, Cheng X and Wang C: Antifatigue and antihypoxia activities of oligosaccharides and polysaccharides from Codonopsis pilosula in mice. Food Funct 11: 6352-6362, 2020.

12. Gao SM, Liu JS, Wang M, Cao TT, Qi YD, Zhang BG, Sun XB, Liu HT and Xiao PG: Traditional uses, phytochemistry, pharmacology and toxicology of Codonopsis: A review. J Ethnopharmacol 219: 50-70, 2018.

13. Yoon IS and Cho SS: Effects of lobetyolin on xanthine oxidase activity in vitro and in vivo: Weak and mixed inhibition. Nat Prod Res: May 29, 2019 (Epub ahead of print). doi: 10.1080/1478 6419.2019.1622108.

14. Deng X, Fu Y, Luo S, Luo X, Wang Q, Hu M, Ma F, Ma CW and Zhou L: Polysaccharide from radix codonopsis has beneficial effects on the maintenance of T-cell balance in mice. Biomed Pharmacother 112: 108682, 2019.
15. Li J, Zhang X, Cao L, Ji J and Gao J: Three inulin-type fructans from Codonopsis pilosula (Franch.) nannf. roots and their prebiotic activity on Bifidobacterium longum. Molecules 23: 3123, 2018.

16. Li J, Wang T, Zhu Z, Yang F, Cao L and Gao J: Structure features and anti-gastric ulcer effects of inulin-type fructan CP-A from the roots of Codonopsis pilosula (Franch.) nannf. Molecules 22: 2258, 2017.

17. Li L, Zhang L and Yang CC: Multi-target strategy and experimental studies of traditional Chinese medicine for Alzheimer's disease therapy. Curr Top Med Chem 16: 537-548, 2016.

18. Tian SS, Yang J, Zhao J and Zhang WD: Application of network biology on study of traditional Chinese medicine. Zhongguo Zhong Yao Za Zhi 43: 274-280, 2018 (In Chinese).

19. Xu H, He L, Chen J, Hou X, Fan F, Wu H, Zhu H and Guo Y: Different types of effective fractions from Radix Isatidis revealed a multiple-target synergy effect against respiratory syncytial virus through RIG-I and MDA5 signaling pathways, a pilot study to testify the theory of superposition of traditional Chinese Medicine efficacy. J Ethnopharmacol 239: 111901, 2019.

20. Grogan KE and Perry GH: Studying human and nonhuman primate evolutionary biology with powerful in vitro and in vivo functional genomics tools. Evol Anthropol 29: 143-158, 2020.

21. Park HW and Weiss ST: Understanding the molecular mechanisms of asthma through transcriptomics. Allergy Asthma Immunol Res 12: 399-411, 2020.

22. Kazimierczyk M,Kasprowicz MK,Kasprzyk ME and Wrzesinski J: Human long noncoding RNA interactome: Detection, characterization and function. Int J Mol Sci 21: 1027, 2020.

23. Tong H, Mao D, Zhai M,Zhang Z, Sun G and Jiang G: Macrophage activation induced by the polysaccharides isolated from the roots of Sanguisorba officinalis. Pharm Biol 53: 1511-1515, 2015.

24. Chen F, Huang G, Yang Z and Hou Y: Antioxidant activity of Momordica charantia polysaccharide and its derivatives. Int J Biol Macromol 138: 673-680, 2019.

25. Sun K, Yang P, Zhao R, Bai Y and Guo Z: Matrine attenuates D-Galactose-induced aging-related behavior in mice via inhibition of cellular senescence and oxidative stress. Oxid Med Cell Longev 2018: 7108604, 2018.

26. Du HM, Wang YJ, Liu X, Wang SL, Wu SM, Yuan Z and Zhu XK: Defective central immune tolerance induced by high-dose D-Galactose resembles aging. Biochemistry (Mosc) 84: 617-626, 2019.

27. Liu J, Chen D, Wang Z, Chen C, Ning D and Zhao S: Protective effect of walnut on d-galactose-induced aging mouse model. Food Sci Nutr 3: 969-976, 2019.

28. Fang JY, Du YQ, Liu WZ, Ren JL, Li YQ, Chen XY, Lv NH, Chen YX and Lv B; Chinese Society of Gastroenterology, Chinese Medical Association: Chinese consensus on chronic gastritis (2017, Shanghai). J Dig Dis 19: 182-203, 2018.

29. Livak KJ and Schmittgen TD: Analysis of relative gene expression data using real-time quantitative PCR and the 2(-Delta Delta C(T)) method. Methods 25: 402-408, 2001.

30. Soenen S and Chapman IM: Body weight, anorexia, and undernutrition in older people. J Am Med Dir Assoc 14: 642-648, 2013.

31. Fulop T, Witkowski JM, Pawelec G, Alan C and Larbi A: On the immunological theory of aging. Interdiscip Top Gerontol 39: 163-176, 2014.

32. Martínez de Toda I, Vida C, Sanz San Miguel L and De la Fuente M: Function, oxidative, and inflammatory stress parameters in immune cells as predictive markers of lifespan throughout aging. Oxid Med Cell Longev 2019: 4574276, 2019.

33. Zhao X, Yi R, Zhou X, Mu J, Long X, Pan Y, Song JL and Park KY: Preventive effect of Lactobacillus plantarum KSFY02 isolated from naturally fermented yogurt from Xinjiang, China, on d-galactose-induced oxidative aging in mice. J Dairy Sci 102: 5899-5912, 2019.

34. Tarnawski AS, Ahluwalia A and Jones MK: Increased susceptibility of aging gastric mucosa to injury: The mechanisms and clinical implications. World J Gastroenterol 20: 4467-4482, 2014.

35. Dinis-Ribeiro $M$ and Kuipers EJ: Identification of gastric atrophic changes: From histopathology to endoscopy. Endoscopy 47: 533-537, 2015.

36. Ren WY, Wu KF, Li X, Luo M, Liu HC, Zhang SC and Hu Y: Age-related changes in small intestinal mucosa epithelium architecture and epithelial tight junction in rat models. Aging Clin Exp Res 26: 183-191, 2014.

37. Hou P, Zhou X, Yu L, Yao Y, Zhang Y, Huang Y, Chen M, Yi L and Mi M: Exhaustive exercise induces gastrointestinal syndrome through reduced ILC3 and IL-22 in mouse model. Med Sci Sports Exerc 52: 1710-1718, 2020. 
38. Clark R and Johnson R: Malabsorption syndromes. Nurs Clin North Am 53: 361-374, 2018.

39. Mansoori B, Rogiewicz A and Slominski BA: The effect of canola meal tannins on the intestinal absorption capacity of broilers using a D-xylose test. J Anim Physiol Anim Nutr (Berl) 99: 1084-1093, 2015.

40. Oteiza PI, Fraga CG, Mills DA and Taft DH: Flavonoids and the gastrointestinal tract: Local and systemic effects. Mol Aspects Med 61: 41-49, 2018.

41. Deng Y, Han X, Tang S, Li C, Xiao W and Tan Z: Magnolol and Honokiol attenuate apoptosis of enterotoxigenic escherichia coli-induced intestinal epithelium by maintaining secretion and absorption homeostasis and protecting mucosal integrity. Med Sci Monit 24: 3348-3356, 2018

42. Kitazawa T, Yoshida A, Tamano T, Teraoka $\mathrm{H}$ and Kaiya $\mathrm{H}$ : Age-dependent reduction of ghrelin- and motilin-induced contractile activity in the chicken gastrointestinal tract Peptides 43: 88-95, 2013.

43. Ivic I, Solymar M, Fulop BD, Hashimoto H, Toth G, Tamas A Juhasz T, Koller A and Reglodi D: Aging-induced modulation of pituitary adenylate cyclase-activating peptide- and vasoactive intestinal peptide-induced vasomotor responses in the arteries of mice. J Vasc Res 54: 359-366, 2017.

44. Korkmaz OT, Ay H, Aytan N, Carreras I, Kowall NW, Dedeoglu A and Tuncel N: Vasoactive intestinal peptide decreases $\beta$-amyloid accumulation and prevents brain atrophy in the 5xFAD mouse model of Alzheimer's disease. J Mol Neurosci 68: 389-396, 2019.

45. Planes-Muñoz D, López-Nicolás R, González-Bermúdez CA Ros-Berruezo $\mathrm{G}$ and Frontela-Saseta C: In vitro effect of green tea and turmeric extracts on GLP-1 and CCK secretion: The effect of gastrointestinal digestion. Food Funct 9: 5245-5250, 2018.

46. Hollander D, Tarnawski A, Stachura J and Gergely $\mathrm{H}$ : Morphologic changes in gastric mucosa of aging rats. Dig Dis Sci 34: 1692-1700, 1989.

47. Takeda K, Okumura T, Taniguchi K and Adachi-Yamada T: Adult intestine aging model. Adv Exp Med Biol 1076: 11-23, 2018.

48. Tan BK, Chen J, Hu J, Amar O, Mattu HS, Ramanjaneya M, Patel V, Lehnert H and Randeva HS: Circulatory changes of the novel adipokine adipolin/CTRP12 in response to metformin treatment and an oral glucose challenge in humans. Clin Endocrinol (Oxf) 81: 841-846, 2014.
49. Chen H, Ma X, Liu Y, Ma L, Chen Z, Lin X, Si L, Ma X and Chen X: Gut microbiota interventions with clostridium butyricum and norfloxacin modulate immune response in experimental autoimmune encephalomyelitis mice. Front Immunol 10: 1662, 2019.

50. Castañeda-Delgado JE, Frausto-Lujan I, González-Curiel I, Montoya-Rosales A, Serrano CJ, Torres-Juarez F, Enciso-Moreno JA and Rivas-Santiago B: Differences in cytokine production during aging and its relationship with antimicrobial peptides production. Immunol Invest 46: 48-58, 2017.

51. Vemuri R, Shinde T, Gundamaraju R, Gondalia SV, Karpe AV, Beale DJ, Martoni CJ and Eri R: Lactobacillus acidophilus DDS-1 modulates the gut microbiota and improves metabolic profiles in aging mice. Nutrients 10: 1255, 2018.

52. Brasili E, Mengheri E, Tomassini A, Capuani G, Roselli M, Finamore A, Sciubba F, Marini F and Miccheli A: Lactobacillus acidophilus La5 and Bifidobacterium lactis Bb12 induce different age-related metabolic profiles revealed by $1 \mathrm{H}-\mathrm{NMR}$ spectroscopy in urine and feces of mice. J Nutr 143: 1549-1557, 2013.

53. Westfall S, Lomis $\mathrm{N}$ and Prakash S: Longevity extension in Drosophila through gut-brain communication. Sci Rep 8: 8362, 2018.

54. Fu YP, Feng B, Zhu ZK, Feng X, Chen SF, Li LX, Yin ZQ, Huang C, Chen XF, Zhang BZ, et al: The polysaccharides from Codonopsis pilosula modulates the immunity and intestinal microbiota of cyclophosphamide-treated immunosuppressed mice. Molecules 23: 1801, 2018.

55. Jing Y, Li A, Liu Z, Yang P, Wei J, Chen X, Zhao T, Bai Y, Zha L and Zhang C: Absorption Codonopsis pilosula saponins by coexisting polysaccharides alleviates gut microbial dysbiosis with dextran sulfate sodium-induced colitis in model mice. Biomed Res Int 2018: 1781036, 2018.

This work is licensed under a Creative Commons Attribution-NonCommercial-NoDerivatives 4.0 International (CC BY-NC-ND 4.0) License. 